\title{
Bivariate genome-wide scan for metabolic phenotypes in non-diabetic Chinese individuals from the Stanford, Asia and Pacific Program of Hypertension and Insulin Resistance Family Study
}

\author{
Y.-F. Chiu • L.-M. Chuang • H.-Y. Kao • L.-T. Ho • \\ C.-T. Ting • Y.-J. Hung $\cdot$ Y.-D. Chen $\cdot$ T. Donlon • \\ J. D. Curb • T. Quertermous • C. A. Hsiung • \\ The SAPPHIRe Study Group
}

Received: 13 March 2007 / Accepted: 2 May 2007 / Published online: 20 June 2007

(C) Springer-Verlag 2007

\begin{abstract}
Aims/hypothesis Hypertension, obesity, impaired glucose tolerance and dyslipidaemia are metabolic abnormalities that often cluster together more often than expected by chance alone. Since these metabolic variables are highly heritable and are at least partially genetically determined, the clustering of defects in these traits implies that pleiotropic effects, where a common set of genes influences more than one trait simultaneously, are likely.
\end{abstract}

Y.-F. Chiu and L.-M. Chuang contributed equally to this study.

Y.-F. Chiu • H.-Y. Kao • C. A. Hsiung $(\bowtie)$

Division of Biostatistics and Bioinformatics,

National Health Research Institutes,

35, Keyan Rd,

Zhunan Town, Miaoli Country 350 Taiwan,

Republic of China

e-mail: hsiung@nhri.org.tw

L.-M. Chuang

Department of Internal Medicine,

National Taiwan University Hospital,

Taipei, Taiwan

L.-M. Chuang

Graduate Institute of Preventive Medicine,

National Taiwan University School of Public Health,

Taipei, Taiwan

L.-T. Ho

Department of Medical Research and Education,

Taipei Veterans General Hospital,

Taipei, Taiwan

C.-T. Ting

Division of Interventional Cardiology, Cardiovascular Center,

Taichung Veterans General Hospital,

Taichung, Taiwan
Methods We conducted bivariate linkage analyses for highly correlated traits, aiming to dissect the genetic architecture affecting these traits, in 411 Chinese families participating in the Stanford Asia-Pacific Program of Hypertension and Insulin Resistance Study.

Results We confirmed the pleiotropic effects of the locus at $37 \mathrm{cM}$ on chromosome 20 on the following pairs: (1) fasting insulin and insulin AUC (empirical $p=0.0006$ ); (2) fasting insulin and homeostasis model assessment of

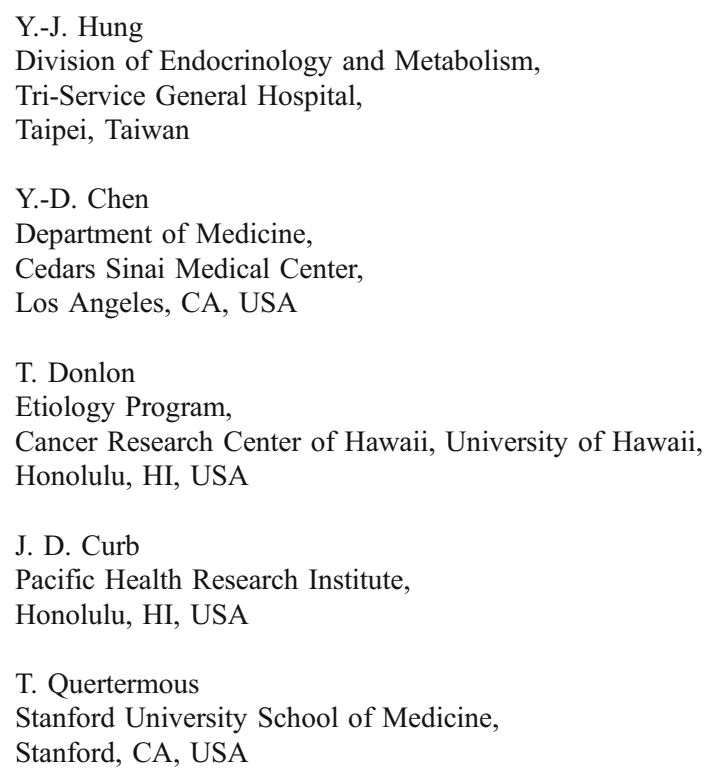


beta cell function (HOMA-beta) (empirical $p=0.0051$ ); and (3) HOMA of insulin resistance (IR) and HOMA-beta (empirical $p=0.0044$ ). In addition, the peak logarithm of the odds (LOD) scores of linkage between a chromosomal locus and a trait for the pair fasting insulin and HOMA-IR rose to 5.10 (equivalent LOD score in univariate analysis, $\operatorname{LOD}_{[1]}=4.01$, empirical $p=8.0 \times 10^{-5}$ ) from 3.67 and 3.42 respectively for these two traits in univariate analysis. Additional significant linkage evidence, not shown in single-trait analysis, was identified at $45 \mathrm{cM}$ on chromosome 16 for the pair $1 \mathrm{~h}$ insulin and the AUC for insulin, with a LOD score of 4.29 (or $\operatorname{LOD}_{[1]}=3.27$, empirical $p=2.0 \times 10^{-4}$ ). This new locus is also likely to harbour the common genes regulating these two traits $\left(p=1.73 \times 10^{-6}\right)$.

Conclusions/interpretation These data help provide a better understanding of the genomic structure underlying the metabolic syndrome.

Keywords Bivariate linkage - Chinese .

Co-incident linkage · Genetic pleiotropy · LOD scores ·

Metabolic syndrome - Quantitative trait locus · QTL .

Variance component method

\begin{tabular}{|c|c|}
\hline Abbreviatior & \\
\hline HOMA-beta & $\begin{array}{l}\text { homeostasis model assessment of beta } \\
\text { cell function }\end{array}$ \\
\hline HOMA-IR & $\begin{array}{l}\text { homeostasis model assessment of } \\
\text { insulin resistance }\end{array}$ \\
\hline LOD & logarithm of the odds \\
\hline QTL & quantitative trait locus \\
\hline SAPPHIRe & $\begin{array}{l}\text { Stanford, Asia and Pacific Program } \\
\text { of Hypertension and Insulin Resistance }\end{array}$ \\
\hline
\end{tabular}

\section{Introduction}

Metabolic syndrome, also known as the insulin resistance syndrome, a cluster of metabolic disorders including insulin resistance, glucose intolerance, hypertension, dyslipidaemia and obesity, is associated with a high risk of developing cardiovascular diseases and type 2 diabetes, as well as with increased all-cause mortality [1-3]. It is known that components of the metabolic syndrome coexist more often than expected by chance alone [4-6]. The clustering of these metabolic traits along with their heritable features suggests that defects in one or more genes may contribute to metabolic syndrome.

Linkage analysis has been widely adopted to narrow down the broad chromosomal regions harbouring susceptibility loci and to elucidate genetic features of various metabolic phenotypes, as well as to categorically define metabolic syndrome [7-9]. The results, however, are mostly limited to identifying: (1) regions with logarithm of the odds (LOD) scores of linkage between a chromosomal locus and a trait that are of merely marginal significance; or (2) regions with modest contributions to overall trait variation [10]. Several attempts have been made to increase the statistical power of quantitative trait locus (QTL) detection, including the use of composite metabolic syndrome traits [11] and meta-analysis; however, both approaches suffer from the presence of genetic and environmental heterogeneity $[12,13]$. Recently, principal component analysis has been applied to associate different 'latent' factors with different grouped metabolic variables, with the latent factors then being used as new phenotypes in the subsequent genome-wide linkage. New interesting loci, not revealed from the original individual phenotypes in linkage analysis, were detected [14-16]. However, the biological nature of the latent factors is not clear.

We have previously shown in the Stanford, Asia and Pacific Program of Hypertension and Insulin Resistance (SAPPHIRe) study that the metabolic phenotypes are inheritable and highly correlated [17, 18]; in addition, we have localised a few susceptibility loci for multiple metabolic phenotypes in the Chinese population [9]. In the present study, we applied bivariate linkage analysis, which could provide improved power over univariate analysis $[15,19]$, to decipher the genetic architecture of the multiple metabolic phenotypes by examining the pleiotropic effects of the mapped loci for pairwise metabolic traits.

\section{Methods}

The SAPPHIRe study The SAPPHIRe study was designed to investigate susceptibility genes for hypertension [17] and insulin resistance in selected Chinese and Japanese populations $[17,20]$. The details of recruitment, exclusion criteria, phenotyping and genotyping have been described elsewhere [17, 20, 21]. In brief, the study was composed of concordant siblings (all siblings with hypertension) and discordant siblings (at least one hypertensive sibling). Probands were recruited on the basis of the following: age at onset 35-60 years or age $>60$ years, but with documentation of hypertension status prior to age 60 available. A total of 2,525 subjects of Japanese or Chinese descent were recruited from centres at San Francisco, Hawaii and Taiwan. All of the subjects underwent a clinical and fasting laboratory examination, with written informed consent obtained prior to examination. Diabetic individuals diagnosed on the basis of the WHO criteria as a result of SAPPHIRe laboratory work or those previously diagnosed were excluded from our analyses, as they usually have abnormal traits measures. Since the number of Japanese in this study was very limited (352 in total) and it's helpful to study a genetically homogeneous population, we focused on the Chinese population in the previous and present 
studies. In addition, 32 subjects taking lipid-lowering medicine were excluded from our analyses. To identify non-paternities, we used 'sib_kin', a module of ASPEX (available from http://aspex.sourceforge.net/, last accessed in May 2007). When non-paternity was identified, that individual was excluded from the analysis [22]. Altogether, 1,365 non-diabetic Chinese subjects (118 parents and 1,247 siblings) with genotyping data from 411 nuclear families were included in this study. The numbers of families with 1 to 8 siblings are $29,138,122,63,37,16,5$ and 1 respectively. The study was approved by the institutional review boards at all participating sites.

Genotyping Details about genotyping procedures were described in our previous study [9]. The genotyping procedure used 376 autosomal markers representing short tandem repeat polymorphisms and yielded an average map density of $10 \mathrm{cM}$. Genotyping quality was monitored by typing 30 samples in duplicate. An error rate of $\sim 1 \%$ was estimated on the basis of these duplicate samples. A published sex-averaged genetic linkage map available from the Marshfield Website (available from http://research. marshfieldclinic.org/genetics, last accessed in May 2007) was used for linkage analysis, the marker order has been confirmed based on a recent release from this site, see Ranade et al. [22] for additional details. ASPEX software was used to examine Mendelian inconsistencies. In the situations where an error was found, the marker data were converted to missing; less than $1 \%$ of the marker data were converted to missing.

Phenotyping After an 8 to $10 \mathrm{~h}$ overnight fast, the participants underwent anthropometric measurements at 08:00 hours without wearing shoes and heavy clothes. Each subject took a $75 \mathrm{~g}$ OGTT after the anthropometric measurements. Fasting blood samples were collected for the measurements of plasma glucose, insulin, total cholesterol, triacylglycerol and LDL-, VLDL- and HDL-cholesterol levels. Then, $75 \mathrm{~g}$ glucose monohydrate (in $300 \mathrm{ml}$ water) was administered to the subject to drink over $5 \mathrm{~min}$. Blood samples were taken for plasma glucose and insulin 1 and $2 \mathrm{~h}$ after glucose loading. The patients were not allowed to eat or drink until the end of the test [21].

Phenotyping procedures for plasma glucose and insulin were as detailed in Ranade et al. [21]. Derivations from the homeostasis model assessment of insulin resistance (HOMA-IR) and beta cell function (HOMA-beta), AUC glucose and AUC insulin were identical to the previous study [9]. Log transformations or Box-Cox transformations were applied to make the conditional distributions (conditional on the covariates) of the phenotypes more nearnormal, as in the previous study [9]. The coefficients of kurtosis for all the traits are less than 2, which appeared not to induce grossly inflated type I error [23].
Covariates We adjusted for the same set of covariates which were adjusted for in the univariate analysis reported previously [9], including BMI, smoking, physical activity, alcohol consumption, field centre and hypertension medication. BMI was calculated as weight in kilograms divided by the square of height in metres $\left(\mathrm{kg} / \mathrm{m}^{2}\right)$. Smoking was classified by four categories: non-smokers, ex-smokers, current light smoker (at least 1, but less than 15 cigarettes per day) and current heavy smoker (at least 15 cigarettes per day). Physical activity was an indicator variable categorising whether the proportion of hours spent on sedentary activity to daily non-basal activities (calculated as $24 \mathrm{~h}$ minus hours of basal activity, including sleeping or lying down) was greater than 0.5 or not. Alcohol consumption was categorised as non-drinker, light drinker, moderate drinker and heavy drinker according to daily average consumption in ounces of alcohol [21]. The recruitment centres included Taiwan, San Francisco and Hawaii. The hypertensive medications possibly affecting plasma and glucose levels [24] included ACE inhibitors and angiotensin receptor blockades. To adjust for these factors, indicator variables were created for all the categorical covariates. Two indicator variables were created to indicate the effects from taking ACE inhibitors and angiotensin receptor blockades, compared with those from not taking these two medications, for the phenotypes related to plasma and glucose levels. Two indicator variables were created for the San Francisco and Hawaii centres respectively, their effects were assessed relatively to the Taiwan centre (baseline). The baseline categories for physical activities, smoker and alcohol consumption were non-basal activities, non-smokers, non-drinking respectively. These covariates were considered to be possible confounders and were all included in these analyses regardless of their significances.

Genome-wide bivariate multipoint linkage analyses The univariate variance component method [25] decomposes the variability of a trait into components for a QTL, the residual polygenic component and the random environmental component [26], which has been extended to a multivariate framework [27]. In the bivariate model, the additive genetic $\left(\rho_{\mathrm{g}}\right)$ and environmental $\left(\rho_{\mathrm{e}}\right)$ correlations between the two traits represent the effects of shared genes or pleiotropy and shared environmental factors, respectively on the phenotypic variance in a trait. The phenotypic correlations $\left(\rho_{\mathrm{p}}\right)$ can be derived from the equation

$\rho_{\mathrm{p}}=\rho_{\mathrm{g}} \sqrt{h_{a}^{2}} \sqrt{h_{b}^{2}}+\rho_{\mathrm{e}} \sqrt{1-h_{a}^{2}} \sqrt{1-h_{b}^{2}}$

where $h_{i}^{2}$ is the heritability for trait $i$ and $i=a, b$, representing either trait 1 or 2 . We conducted a series of bivariate quantitative genetic analyses for all pairwise combinations of metabolic traits using the bivariate approach implemented 
in SOLAR 2.0 (available from http://www.sfbr.org/solar, last accessed in May 2007).

We obtained trait-specific estimates of the variance components due to the QTL, residual genetic factors and environmental factors, respectively, as well as the three associated correlations $\rho_{\mathrm{q}}, \rho_{\mathrm{g}}$ and $\rho_{\mathrm{e}}$, where $\rho_{\mathrm{q}}$ is a measure of shared major gene effects at the QTL where linkage is being assessed. Under this model, we tested the null hypothesis that the variance component attributed from the QTL equals zero for both traits by comparing the likelihood of this restricted model to that of a model in which this variance component was estimated for the traits. Twice the difference in log likelihoods of these models yields a test statistic that is asymptotically distributed as a mixture of $1 / 4 \chi_{0}^{2}, 1 / 2 \chi_{1}^{2}$ and $1 / 4 \chi_{3}^{2}$ [27]. Bivariate linkage analyses were performed for each pairwise combination of metabolic traits. LOD scores obtained from bivariate linkage analyses were also converted to a $1 / 2 \chi_{1}^{2}$ of equivalent $p$ values in univariate linkage analyses, hereafter denoted by $\operatorname{LOD}_{[1]}$. To achieve levels of statistical significance comparable to the LOD score thresholds required in the univariate linkage analyses, we considered bivariate multipoint LOD scores $\geq 4.0$ to be statistically significant evidence of linkage $(p \leq 0.0001), \geq 2.87$ as suggestive evidence $(p \leq 0.001)$ and $\geq 2.06$ as tentative evidence of linkage $(p \leq 0.007)$ [28]. However, only regions showing significant or suggestive evidence of linkage are reported here.

To test pleiotropy and co-incident linkage, likelihoods for a linkage model in which $\rho_{\mathrm{q}}$ was estimated were compared with models in which $\rho_{\mathrm{q}}$ was constrained to 0 (no shared major gene effects in the region, i.e. co-incident linkage) and to 1 or -1 (complete pleiotropy) [29]. In the case of $\rho_{\mathrm{q}}$ being constrained to 0 , the difference between these likelihoods is distributed as a chi-square with 1 degree of freedom. When $\rho_{\mathrm{q}}$ is constrained to 1 or -1 , a boundary, the difference in likelihoods, is distributed as a 1/2:1/2 mixture of $\chi_{1}^{2}$ and a point mass at 0 [27]. The hypothesis of $\rho_{\mathrm{q}}=1$ or -1 (complete pleiotropy) or the hypothesis of $\rho_{\mathrm{q}}=0$ (co-incident linkage) was rejected when $p$ was less than 0.05 .

\section{Results}

Descriptive statistics for demographic characters and eighteen metabolic phenotypes used in bivariate analyses are summarised in Table 1. The genetic, environmental and phenotypic correlations ( \pm standard errors) between selected pairs of traits are reported in Table 2, with regions showing suggestive or significant evidence. Regions with peak LOD scores $\geq 2.87$ (equivalent to an LOD score of 2.0 in univariate analysis) and the results for testing of QTL
Table 1 The clinical and metabolic characteristics of family members in bivariate analyses

\begin{tabular}{lll}
\hline Trait & $\begin{array}{l}\text { Number } \\
\text { of members }\end{array}$ & $\begin{array}{l}\text { Mean } \pm \text { SD } \\
\text { or } \%\end{array}$ \\
\hline Female & 649 & 55.9 \\
Age (years) & 1,161 & $50.12 \pm 10.92$ \\
Fasting glucose (mmol/l) & 1,156 & $4.93 \pm 0.58$ \\
1 h glucose $(\mathrm{mmol} / \mathrm{l})$ & 1,029 & $9.34 \pm 2.22$ \\
2 h glucose $(\mathrm{mmol} / \mathrm{l})$ & 1,002 & $7.20 \pm 1.80$ \\
Fasting insulin $(\mathrm{pmol} / \mathrm{l})$ & 1,153 & $52.67 \pm 34.97$ \\
1 h insulin $(\mathrm{pmol} / \mathrm{l})$ & 1,023 & $548.20 \pm 408.14$ \\
2 h insulin $(\mathrm{pmol} / \mathrm{l})$ & 1,021 & $443.08 \pm 410.09$ \\
HOMA-IR & 1,152 & $1.65 \pm 1.21$ \\
HOMA-beta & 1,152 & $108.04 \pm 155.72$ \\
AUC glucose $(\mathrm{mmol} / \mathrm{l} \times \mathrm{h})$ & 998 & $16.62 \pm 3.10$ \\
AUC insulin $(\mathrm{pmol} / \mathrm{l} \times \mathrm{h})$ & 1,014 & $796.78 \pm 572.45$ \\
Triacylglycerols $(\mathrm{mmol} / \mathrm{l})$ & 1,148 & $1.36 \pm 0.84$ \\
HDL-cholesterol $(\mathrm{mmol} / \mathrm{l})$ & 1,152 & $1.14 \pm 0.33$ \\
LDL-cholesterol $(\mathrm{mmol} / \mathrm{l})$ & 1,134 & $3.03 \pm 0.94$ \\
VLDL-cholesterol (mmol/l) & 1,139 & $0.61 \pm 0.33$ \\
Total cholesterol $(\mathrm{mmol} / \mathrm{l})$ & 1,156 & $4.77 \pm 0.99$ \\
Systolic BP $(\mathrm{mmHg})$ & 1,033 & $127.53 \pm 24.75$ \\
Diastolic BP $(\mathrm{mmHg})$ & 1,033 & $76.41 \pm 13.58$ \\
BMI $\left(\mathrm{kg} / \mathrm{m}^{2}\right)$ & 887 & $25.02 \pm 3.27$ \\
\hline
\end{tabular}

pleiotropy were performed for these traits and are reported in Tables 3 and 4.

The univariate and bivariate linkage profiles are summarised in Fig. 1. In bivariate linkage analyses, when fasting insulin was paired up with HOMA-IR $\left(\operatorname{LOD}_{[1]}=\right.$ 4.01 , empirical $p=0.00008)$, fasting glucose $\left(\operatorname{LOD}_{[1]}=3.09\right.$, empirical $p=0.0002)$, AUC insulin $\left(\operatorname{LOD}_{[1]}=2.63\right.$, empirical $p=0.0006)$, HOMA-beta $\left(\operatorname{LOD}_{[1]}=2.81\right.$, empirical $p=0.0051)$ or $\mathrm{BMI}\left(\mathrm{LOD}_{[1]}=2.82\right.$, empirical $\left.p=0.00032\right)$, the peak of LOD scores remained located at $37 \mathrm{cM}$ on chromosome 20, the same QTL identified from the univariate analyses for fasting insulin, HOMA-IR and HOMA-beta [9]. This QTL was also found for the trait pairs HOMA-IR/ HOMA-beta $\left(\operatorname{LOD}_{[1]}=2.62\right.$, empirical $\left.p=0.0044\right)$ and HOMA-IR/BMI $\left(\operatorname{LOD}_{[1]}=2.61\right.$, empirical $\left.p=0.00028\right)$. The complete pleiotropic effects were confirmed for the pairs fasting insulin/AUC insulin, fasting insulin/HOMAbeta and HOMA-IR/HOMA-beta (Table 4), where the hypothesis of co-incident linkage was rejected $(p<0.05)$ and the hypothesis of pleiotropy linkage was not rejected $(p>0.05)$. In contrast, the correlations between these pairs of metabolic variables under a polygenic model are mostly significantly away from zero, except for the pairs BMI/ fasting insulin or BMI/HOMA-IR (Table 2). The hypotheses of complete co-incident linkage and pleiotropy were both rejected for the highly correlated trait-pair fasting insulin/HOMA-IR, indicating that the common genetic effect may be modified by environmental influences or the effect of epistasis. 
Table 2 Phenotypic $\left(\rho_{\mathrm{p}}\right)$, genetic $\left(\rho_{\mathrm{g}}\right)$ and environmental $\left(\rho_{\mathrm{e}}\right)$ correlations between selected pairs of metabolic phenotypes

\begin{tabular}{lllll}
\hline Pairs of metabolic traits & & $\rho_{\mathrm{e}}$ & $\rho_{\mathrm{g}}$ & $\rho_{\mathrm{p}}$ \\
\hline Fasting insulin & Fasting glucose & $0.41 \pm 0.1^{*}$ & $0.21 \pm 0.11$ & $0.28 \pm 0.06^{*}$ \\
Fasting insulin & HOMA-beta & $0.83 \pm 0.04^{*}$ & $0.49 \pm 0.09^{*}$ & $0.66 \pm 0.11^{*}$ \\
Fasting insulin & HOMA-IR & $0.99 \pm 0.0023 *$ & $0.96 \pm 0.01^{*}$ & $0.98 \pm 0.14^{*}$ \\
Fasting insulin & AUC insulin & $0.55 \pm 0.06^{*}$ & $0.66 \pm 0.09^{*}$ & $0.59 \pm 0.07^{*}$ \\
Fasting insulin & BMI & $0.14 \pm 0.09$ & $-0.2 \pm 0.14$ & $0.0021 \pm 0.03$ \\
Fasting glucose & SBPc & $-0.00017 \pm 0.11$ & $-0.1 \pm 0.14$ & $-0.04 \pm 0.03$ \\
HOMA-IR & HOMA-beta & $0.78 \pm 0.06^{*}$ & $0.23 \pm 0.12$ & $0.51 \pm 0.10^{*}$ \\
HOMA-IR & BMI & $0.09 \pm 0.09$ & $-0.13 \pm 0.14$ & $0.0017 \pm 0.03$ \\
Systolic BP & Diastolic BP & $0.75 \pm 0.03^{*}$ & $0.51 \pm 0.22^{*}$ & $0.7 \pm 0.087^{*}$ \\
1 h insulin & AUC insulin & $0.95 \pm 0.01^{*}$ & $0.96 \pm 0.02^{*}$ & $0.94 \pm 0.14^{*}$ \\
1 h insulin & HDL-cholesterol & $-0.02 \pm 0.1$ & $-0.29 \pm 0.12^{*}$ & $-0.15 \pm 0.04^{*}$ \\
AUC insulin & HDL-cholesterol & $-0.05 \pm 0.11$ & $-0.3 \pm 0.11^{*}$ & $-0.18 \pm 0.03^{*}$ \\
HDL-cholesterol & VLDL-cholesterol & $-0.24 \pm 0.11^{*}$ & $-0.44 \pm 0.09^{*}$ & $-0.35 \pm 0.04^{*}$ \\
HDL-cholesterol & Triacylglycerols & $-0.27 \pm 0.11^{*}$ & $-0.43 \pm 0.09^{*}$ & $-0.36 \pm 0.05^{*}$ \\
HDL-cholesterol & BMI & $-0.45 \pm 0.12^{*}$ & $0.35 \pm 0.11^{*}$ & $-0.01 \pm 0.05$ \\
2 h insulin & VLDL-cholesterol & $0.08 \pm 0.1$ & $0.5 \pm 0.11^{*}$ & $0.28 \pm 0.03^{*}$ \\
Total cholesterol & LDL-cholesterol & $0.87 \pm 0.02^{*}$ & $0.94 \pm 0.01^{*}$ & $0.91 \pm 0.13^{*}$ \\
\hline
\end{tabular}

Values are means $\pm \mathrm{SE}$

${ }^{*} p<0.05$

In the univariate analyses, we did not identify any susceptibility QTL for systolic or diastolic blood pressure; however, the QTL at $88 \mathrm{cM}$ became noticeable with a $\operatorname{LOD}_{[1]}$ of 2.46 (empirical $p=0.00024$ ) when looking at these two traits simultaneously. A peak at $93 \mathrm{cM}$ in this region also manifested itself when modelling fasting glucose and systolic blood pressure $\left(\operatorname{LOD}_{[1]}=3.12\right.$, empirical $p=0.00004)$ together. However, this evidence of linkage could be caused by co-incident effects, since the hypothesis of complete co-incident linkage was not rejected $(p>0.05)$ in both regions. Although the environmental and genetic correlations between systolic and diastolic blood pressure are as high as $0.75 \pm 0.03$ and $0.51 \pm 0.22$, respectively, these correlations are almost negligible between systolic blood pressure and fasting glucose. The peaks at around $51 \mathrm{cM}$ on chromosome 20 that we observed in univariate analyses for fasting glucose became more significant when combined with systolic blood pressure $\left(\mathrm{LOD}_{[1]}=2.06\right.$, empirical $\left.p=0.0025\right)$ in bivariate analyses.

Several pair-wise combinations with HDL-cholesterol showed similar patterns to HDL-cholesterol with two peaks identified at 113 and $123 \mathrm{cM}$ on chromosome 12 respectively, including the pairs HDL-cholesterol/VLDL-cholesterol, HDL-cholesterol/triacylglycerols, HDL-cholesterol/BMI, HDL-cholesterol/1 $\mathrm{h}$ insulin and HDL-cholesterol/AUC insulin. Their $\mathrm{LOD}_{[1]}$ scores ranged from 2.15 to 3.01 with empirical $p$ values varying from 0.00012 to 0.0018 . These QTLs, however, could have been caused by co-incident effects, as we failed to reject the hypothesis of complete co-incident linkage while the hypothesis of complete pleiotropy was rejected, except for HDL-cholesterol/BMI, HDL- cholesterol/1 $\mathrm{h}$ insulin and HDL-cholesterol/AUC insulin (both hypotheses not rejected; Table 4). The genetic correlations between HDL-cholesterol and these traits are all statistically significant as shown in Table 2. In addition, the linkage signals became more substantial in bivariate analysis than in univariate analysis for the following paired traits: total cholesterol/LDL on chromosome $1 \mathrm{p}$, fasting insulin/fasting glucose on chromosome $2 p$, fasting insulin/ HOMA-beta on chromosome $18 \mathrm{q}$ and $14 \mathrm{q}$ and HOMA-IR/ HOMA-beta on 14q.

A new QLT located at $148 \mathrm{cM}$ on chromosomes 9, but not discovered in the univariate linkage analyses, was identified for the combinations HOMA-IR/HOMA-beta $\left(\mathrm{LOD}_{[1]}=2.22\right.$, empirical $\left.p=0.0083\right)$ and fasting insulin/ HOMA-beta $\left(\operatorname{LOD}_{[1]}=2.47\right.$, empirical $\left.p=0.009\right)$. The peak for $2 \mathrm{~h}$ insulin remained in the same region after combining with VLDL-cholesterol $\left(\operatorname{LOD}_{[1]}=2.02\right.$, empirical $\left.p=0.001\right)$; however, this could be attributed to a co-incident effect on this QTL $(p=0.81)$. Interestingly, the QTL located at $45 \mathrm{cM}$ for $1 \mathrm{~h}$ insulin and AUC insulin on chromosome 16 $\left(\mathrm{LOD}_{[1]}=3.27\right.$, empirical $\left.p=0.0002\right)$ but not identified in the univariate linkage analyses also showed significant evidence of pleiotropic effects (Table 4).

In summary, since AUC insulin was derived from three insulin-related measures and HOMA-beta and HOMA-IR were derived from fasting insulin and fasting glucose, these traits are similar in nature. Linkage signals observed at $37 \mathrm{cM}$ on chromosome 20 from the trait-pairs including fasting insulin/HOMA-beta, fasting insulin/AUC insulin and HOMAIR/HOMA-beta resulted from the effects of pleiotropy. On the other hand, the linkage evidence observed for 
Table 3 Regions with peak LOD scores $\geq 2.87$ in genome-wide bivariate multipoint linkage analysis

\begin{tabular}{|c|c|c|c|c|c|c|}
\hline Trait 1 & Trait 2 & Chromosome & $\mathrm{cM}$ & MLS & $\operatorname{MLS}_{[1]}$ & Empirical $p$ value \\
\hline \multirow[t]{3}{*}{ Fasting insulin } & \multirow[t]{3}{*}{ Fasting glucose } & 2 & 90 & 2.87 & 2.00 & 0.0028 \\
\hline & & 20 & 37 & 4.09 & 3.09 & 0.0002 \\
\hline & & 20 & 73 & 2.89 & 2.022 & 0.0027 \\
\hline \multirow[t]{4}{*}{ Fasting insulin } & \multirow[t]{4}{*}{ HOMA-beta } & 9 & 148 & 3.40 & 2.47 & 0.009 \\
\hline & & 14 & 43 & 3.48 & 2.54 & 0.0084 \\
\hline & & 18 & 55 & 3.05 & 2.16 & 0.014 \\
\hline & & 20 & 37 & 3.78 & 2.81 & 0.0051 \\
\hline \multirow[t]{3}{*}{ Fasting insulin } & \multirow[t]{3}{*}{ HOMA-IR } & 20 & 21 & 3.35 & 2.43 & 0.0038 \\
\hline & & 20 & 28 & 3.65 & 2.69 & 0.0021 \\
\hline & & 20 & 37 & 5.10 & 4.01 & 0.00008 \\
\hline \multirow[t]{2}{*}{ Fasting insulin } & \multirow[t]{2}{*}{ AUC insulin } & 5 & 85 & 3.18 & 2.28 & 0.0016 \\
\hline & & 20 & 37 & 3.58 & 2.63 & 0.0006 \\
\hline Fasting insulin & BMI & 20 & 37 & 3.79 & 2.82 & 0.00032 \\
\hline \multirow[t]{3}{*}{ Fasting glucose } & \multirow[t]{3}{*}{ Systolic BP } & 20 & 51 & 2.93 & 2.06 & 0.00252 \\
\hline & & 20 & 54 & 2.91 & 2.04 & 0.0026 \\
\hline & & 20 & 93 & 4.12 & 3.12 & 0.00004 \\
\hline \multirow[t]{3}{*}{ HOMA-IR } & \multirow[t]{3}{*}{ HOMA-beta } & 9 & 148 & 3.11 & 2.22 & 0.0083 \\
\hline & & 14 & 44 & 3.45 & 2.51 & 0.0052 \\
\hline & & 20 & 37 & 3.57 & 2.62 & 0.0044 \\
\hline HOMA-IR & BMI & 20 & 37 & 3.56 & 2.61 & 0.00028 \\
\hline Systolic BP & Diastolic BP & 20 & 88 & 3.39 & 2.46 & 0.00024 \\
\hline $1 \mathrm{~h}$ insulin & AUC insulin & 16 & 45 & 4.29 & 3.27 & 0.0002 \\
\hline $1 \mathrm{~h}$ insulin & HDL-cholesterol & 12 & 114 & 3.06 & 2.17 & 0.0018 \\
\hline AUC insulin & HDL-cholesterol & 12 & 113 & 3.03 & 2.15 & 0.0014 \\
\hline \multirow[t]{2}{*}{ HDL-cholesterol } & \multirow[t]{2}{*}{ VLDL-cholesterol } & 12 & 113 & 4.01 & 3.01 & 0.00012 \\
\hline & & 12 & 123 & 3.29 & 2.38 & 0.00076 \\
\hline \multirow[t]{2}{*}{ HDL-cholesterol } & \multirow[t]{2}{*}{ Triacylglycerols } & 12 & 113 & 3.72 & 2.76 & 0.0004 \\
\hline & & 12 & 123 & 3.07 & 2.18 & 0.0011 \\
\hline \multirow[t]{2}{*}{ HDL-cholesterol } & \multirow[t]{2}{*}{ BMI } & 12 & 113 & 3.75 & 2.78 & 0.00052 \\
\hline & & 12 & 124 & 3.57 & 2.62 & 0.00064 \\
\hline \multirow[t]{3}{*}{ Adiponectin } & \multirow[t]{3}{*}{ HDL-cholesterol } & 12 & 115 & 3.12 & 2.22 & 0.00068 \\
\hline & & 12 & 126 & 3.29 & 2.37 & 0.00052 \\
\hline & & 15 & 31 & 3.71 & 2.74 & 0.00028 \\
\hline $2 \mathrm{~h}$ insulin & VLDL-cholesterol & 9 & 77 & 2.89 & 2.02 & 0.001 \\
\hline Total cholesterol & LDL-cholesterol & 1 & 71 & 3.30 & 2.38 & 0.00096 \\
\hline
\end{tabular}

$B P$ Blood pressure, $M L S$ maximum LOD score in bivariate linkage analysis, $M L S_{[1]}$ the bivariate LOD score converted to LOD score with equivalent $p$ value in univariate linkage analyses

most of the trait-pairs comprised of different metabolic measures, such as fasting glucose/systolic blood pressure (on 20q13.32), systolic/diastolic blood pressure (on 20q13.31), HDL-cholesterol/VLDL-cholesterol (on 12q21.33-12q22), HDL-cholesterol/triacylglycerols (on 12q21.33-12q22) and $2 \mathrm{~h}$ insulin/VLDL-cholesterol (on 9q13-9q21.1) was mostly due to co-incident effects. BMI doesn't seem to be correlated with fasting insulin or HOMAIR in our study, but it is genetically and environmentally correlated with HDL-cholesterol, with the genetic and environment correction coefficients 'pointing' in the opposite directions. The genetic correlation between BMI and HDL-cholesterol is probably due to the pleiotropy effect from the QTL on chromosome 12 .

\section{Discussion}

In this study, we present evidence that bivariate linkage analyses of inter-related metabolic phenotypes improve the ability to localise susceptibility loci for one trait. We also show that bivariate analyses appear to be able to differentiate between, on the one hand, pleiotropic effects of a single locus influencing all the traits and, on the other hand, separate tightly clustered loci each influencing a single trait, thus facilitating our understanding of genomic structure for the complex nature of metabolic syndrome.

By applying the bivariate linkage approach, we identified a few putative QTLs for multiple inter-related metabolic phenotypes that are clustered on chromosomes 
Table 4 Pleiotropy and co-incident linkage at QTLs identified from the genome-wide bivariate linkage analyses

\begin{tabular}{|c|c|c|c|c|c|c|c|}
\hline \multirow[t]{3}{*}{ Bivariate } & & \multirow[t]{3}{*}{ Chromosome } & \multirow[t]{3}{*}{$\mathrm{cM}$} & \multirow[t]{3}{*}{ MLS } & \multicolumn{3}{|l|}{$p$ value of the test } \\
\hline & & & & & \multirow{2}{*}{$\frac{\text { Co-incident linkage }}{\rho_{\mathrm{q}}=0}$} & \multicolumn{2}{|l|}{ Pleiotropy } \\
\hline & & & & & & $\rho_{\mathrm{q}}=1$ & $\rho_{\mathrm{q}}=-1$ \\
\hline Fasting insulin & Fasting glucose & 20 & 37 & 4.09 & 0.31 & 0.095 & \\
\hline Fasting insulin & HOMA-beta & 20 & 37 & 3.78 & $0.0018^{\mathrm{a}}$ & $0.16^{\mathrm{a}}$ & \\
\hline Fasting insulin & HOMA-IR & 20 & 37 & 5.10 & $6.71 \times 10^{-5}$ & 0.0058 & \\
\hline Fasting insulin & AUC insulin & 20 & 37 & 3.58 & $1.52 \times 10^{-4 a}$ & $0.5^{\mathrm{a}}$ & \\
\hline Fasting insulin & BMI & 20 & 37 & 3.79 & 0.57 & 0.0025 & 0.50 \\
\hline Fasting glucose & SBP & 20 & 93 & 4.12 & $0.38^{\mathrm{b}}$ & $3.61 \times 10^{-4}$ & $0.0095^{\mathrm{b}}$ \\
\hline HOMA-IR & HOMA-beta & 20 & 37 & 3.57 & $0.0030^{\mathrm{a}}$ & $0.14^{\mathrm{a}}$ & \\
\hline HOMA-IR & BMI & 20 & 37 & 3.56 & 0.54 & 0.27 & 0.50 \\
\hline SBP & DBP & 20 & 88 & 3.39 & $0.086^{\mathrm{b}}$ & $0.0055^{\mathrm{b}}$ & \\
\hline $1 \mathrm{~h}$ insulin & AUC insulin & 16 & 45 & 4.29 & $1.73 \times 10^{-6}$ & $2.04 \times 10^{-7}$ & 0.5 \\
\hline $1 \mathrm{~h}$ insulin & HDL-cholesterol & 12 & 114 & 3.06 & $0.36^{\mathrm{b}}$ & $0.036^{\mathrm{b}}$ & 0.39 \\
\hline AUC insulin & HDL-cholesterol & 12 & 113 & 3.03 & $0.32^{\mathrm{b}}$ & $0.0036^{\mathrm{b}}$ & 0.50 \\
\hline HDL-cholesterol & VLDL-cholesterol & 12 & 113 & 4.01 & $0.15^{\mathrm{b}}$ & 0.0058 & $0.012^{\mathrm{b}}$ \\
\hline HDL-cholesterol & Triacylglycerols & 12 & 113 & 3.72 & $0.15^{\mathrm{b}}$ & 0.0092 & $0.020^{\mathrm{b}}$ \\
\hline HDL-cholesterol & BMI & 12 & 113 & 3.75 & $0.54^{\mathrm{b}}$ & 0.15 & $0.0041^{\mathrm{b}}$ \\
\hline $2 \mathrm{~h}$ insulin & VLDL-cholesterol & 9 & 77 & 2.89 & $0.81^{\mathrm{b}}$ & $0.031^{\mathrm{b}}$ & \\
\hline Total cholesterol & LDL-cholesterol & 1 & 71 & 3.30 & 0.0022 & 0.015 & \\
\hline
\end{tabular}

$D B P$ Diastolic blood pressure, $M L S$ maximum LOD score, SBP systolic blood pressure

${ }^{\mathrm{a}}$ Evidence of pleiotropy linkage

${ }^{\mathrm{b}}$ Evidence of co-incident linkage

12 and 20. Additionally, we found significant or suggestive evidence of linkage of 10 QTLs for various metabolic variables scattered on chromosomes $1 p, 2 p, 5 q, 9 q, 14 q$, $16 \mathrm{p}$ and $18 \mathrm{q}$. In general, the LOD scores of these loci were higher than those obtained in univariate linkage analyses, thus substantiating the results showing only weak evidence of linkage in the previous report [9]. We also found new QTLs on chromosome $9\left(\operatorname{LOD}_{[1]}=2.47\right.$ and 2.22 at $148 \mathrm{cM}$
Fig. 1 Total genome review of quantitative trait univariate and bivariate linkage analyses. Results with peak LOD scores $\geq 2.0$ are displayed (LOD scores from bivariate linkage analysis have been converted to LOD scores with equivalent $p$ values in univariate analysis)
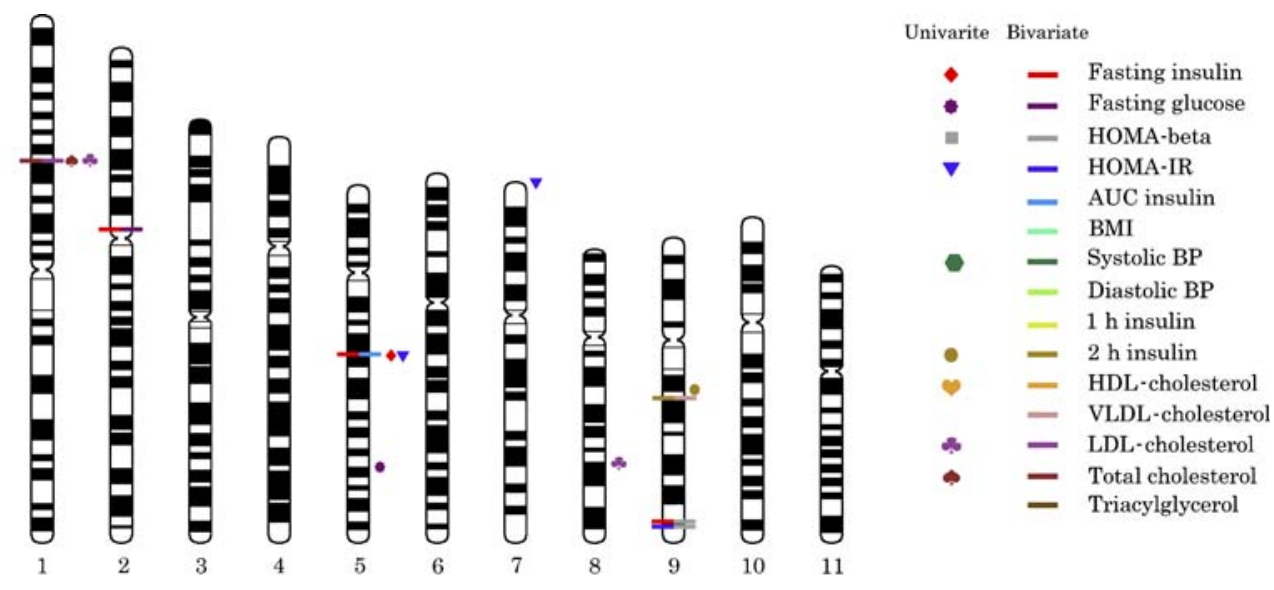

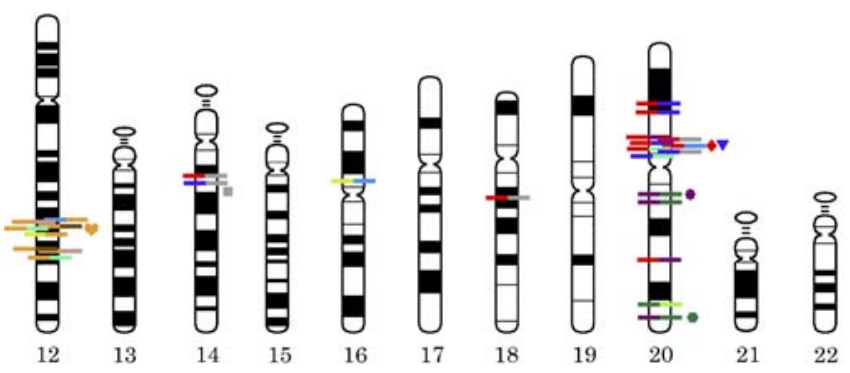


for the trait pairs fasting insulin/HOMA-beta and HOMAIR/HOMA-beta, respectively) and on chromosome 16 $\left(\operatorname{LOD}_{[1]}=3.27\right.$ at $45 \mathrm{cM}$ for $1 \mathrm{~h}$ insulin/AUC insulin) that have not been identified in the univariate linkage analyses. There are roughly 35 QTLs showing only tentative evidence of linkage with $\operatorname{LOD}_{[1]} \geq 2.00$, indicating that bivariate linkage analysis is a more powerful approach than univariate analysis.

The QTL at $71 \mathrm{cM}$ on chromosome 1 identified for total cholesterol $(\mathrm{LOD}=2.16)$ in the univariate analysis became slightly more substantial for the trait pair total cholesterol/ LDL cholesterol $\left(\operatorname{LOD}_{[1]}=2.38\right)$. The susceptibility QTL for fasting insulin, HOMA-IR and HOMA-beta located at $37 \mathrm{cM}$ on chromosome 20 , a region previously identified, was also identified in this study, with LOD scores of 3.78 to 5.10 (or $\mathrm{LOD}_{[1]}$ scores of 2.81-4.01) compared with LODs of 1.71 to 3.65 as estimated in the univariate analysis [9]. Interestingly, in the bivariate analyses, this locus also influenced fasting glucose, AUC insulin and BMI. Although the genetic correlations between fasting insulin and fasting glucose or BMI are relatively low, possible genetic pleiotropy for these metabolic phenotypes was observed. This notion is particularly true for fasting insulin and HOMA-beta or AUC insulin, which are consistent with a hypothesis of complete pleiotropy. As for the interaction between fasting insulin and glucose levels, we cannot exclude the possibility of partial pleiotropy. These findings are consistent with previous studies showing that trait pairs exhibiting low to moderate overall genetic and phenotypic correlations appear more informative for bivariate analyses than pairs with high genetic correlations [19, 27, 30, 31]. On the other hand, the QTL responsible for fasting insulin together with BMI or HOMA-IR could be due to co-incident linkage, suggesting that the susceptibility genes are independently responsible for fasting insulin or BMI and simply happen to cluster together.

The candidate gene proprotein convertase subtilisin/ kexin type 2 (PCSK2), which is located at the region on chromosome 20 , has been reported to be associated with type 2 diabetes in a Japanese study [32]. In addition, it is very likely that other genes nearby also contribute to insulin resistance and/or beta cell functions. We are currently working on the identification of SNPs from other candidate genes in this region.

Surprisingly, the two QTLs that peaked at 113 and $123 \mathrm{cM}$ on chromosome 12 for HDL-cholesterol, VLDLcholesterol and triacylglycerols also showed great influences on $1 \mathrm{~h}$ insulin, AUC insulin and BMI. This QTL was also previously identified by univariate linkage analyses [33]. Since the highest peak loci for different trait pairs were all located at $113 \mathrm{cM}$ from pter, this indicates the possibility that the same gene in this region contributes to different phenotypes. However, bivariate linkage analyses showed that the QTL for the trait pairs HDL-cholesterol/ VLDL-cholesterol and HDL-cholesterol/triacylglycerols could be caused by co-incident linkage. The complete co-incident linkage or pleiotropy hypotheses were not rejected for HDL-cholesterol and BMI. BMI has been shown to have pleiotropic effects with several phenotypes related to insulin resistance syndrome on chromosome $6 \mathrm{q}$ [34], yet the phenomenon was not observed in this study. Although we are not clear about the underlying genetic mechanism, the presence of incomplete pleiotropy could reduce the power to reject co-incident linkage. It is likely that several allelic variants of a major putative gene exert a differential influence on the two correlated phenotypes. Or one genetic effect, modified by environmental factors or by genetic epistasis, influences the two correlated phenotypes. Several candidate genes have been reported in the region identified for HDL-cholesterol on chromosome 12, including sterol $O$-acyltransferase 2 (SOAT2) on 12q13.13, apolipoprotein $\mathrm{F}(A P O F)$ on 12q13.2, nuclear receptor subfamily 1 , group $\mathrm{H}$, member 4 (NR1H4) on 12q23.1, transcription factor 1 (TCF1) on $12 \mathrm{q} 24$ and scavenger receptor class $\mathrm{B}$, member 1 (SCARB1) on $12 \mathrm{q} 24.31$ [35]. When HDL-cholesterol was paired up with either VLDL-cholesterol, triacylglycerols or BMI in the bivariate analysis, the peak was located at $12 \mathrm{q} 23.2$, where the marker PAH closest to the peak has been shown to be a putative QTL with a LOD of 2.13 for HDLcholesterol levels in the Mexican American population in the San Antonio Family Heart Study [36]. In this region, the NR1H4 gene, also known as the FXR gene, is a key regulator of cholesterol homeostasis, as shown in $\mathrm{Fxr}^{-l}$ knockout mice [37].

In search for the genetic loci for metabolic syndrome (as a categorical diagnosis) and its related traits, many studies have been conducted in different populations [11, 14, 16, 38, 39], including Chinese living in Hong Kong [7]. We were unable to replicate the results from that study by again showing evidence of linkage for metabolic syndrome on chromosome 1 at $169.5-181.5 \mathrm{cM}$ and on chromosome 2 at 44.1-57.3 cM. However, we did observe a significant QTL on chromosome 16 at $45 \mathrm{cM}\left(\mathrm{LOD}=4.29\right.$ and $\mathrm{LOD}_{[1]}=3.27$ for $1 \mathrm{~h}$ insulin and AUC insulin), similar to the reported region at $45.2-65.4 \mathrm{cM}$ (with $\mathrm{LOD}=1.75,1.61$ and 1.25 for metabolic syndrome, HOMA-IR and HDL-cholesterol respectively) in Hong Kong Chinese [7].

In conclusion, several metabolic phenotypes related to the complex metabolic syndrome might be attributed to common genetic influences, i.e. genetic pleiotropy, as we have observed for obesity/insulin $(37 \mathrm{cM}$ on chromosome 20) and lipid/insulin (113 cM on chromosome 12) phenotypes. Interestingly, the genes responsible for fasting insulin, HOMA-beta and AUC insulin might be identical and are located at $37 \mathrm{cM}$ from pter on chromo- 
some 20. These data help provide a better understanding of the genetic factors in the chromosomal regions influencing different metabolic traits, thus facilitating the characterisation of the positional candidates that are involved in the pathogenesis of metabolic syndrome in Chinese populations.

Acknowledgements We thank S.-Y. Liu and Y.-J. Tsai for their help with the computing. We thank patients and their families for participating in this study. We also thank D. Paltoo from the National Heart, Lung and Blood Institute and all the other members of the SAPPHIRe project for their help. This work was partially supported by grant BS-095-PP01 from National Health Research Institutes.

Duality of interest None of the authors involved in this study had any duality of interest.

\section{References}

1. Haffner SM, Valdez RA, Hazuda HP, Mitchell BD, Morales PA, Stern MP (1992) Prospective analysis of the insulin-resistance syndrome (syndrome X). Diabetes 41:715-722

2. Hanson RL, Imperatore G, Bennett PH, Knowler WC (2002) Components of the 'metabolic syndrome' and incidence of type 2 diabetes. Diabetes 51:3120-3127

3. Lakka HM, Laaksonen DE, Lakka TA et al (2002) The metabolic syndrome and total and cardiovascular disease mortality in middle-aged men. JAMA 288:2709-2716

4. Smoak CG, Burke GL, Webber LS, Harsha DW, Srinivasan SR, Berenson GS (1987) Relation of obesity to clustering of cardiovascular disease risk factors in children and young adults. The Bogalusa Heart Study. Am J Epidemiol 125:364-372

5. Reaven GM (1988) Banting lecture 1988. Role of insulin resistance in human disease. Diabetes 37:1595-1607

6. Schmidt MI, Watson RL, Duncan BB et al (1996) Clustering of dyslipidemia, hyperuricemia, diabetes, and hypertension and its association with fasting insulin and central and overall obesity in a general population. Atherosclerosis Risk in Communities Study Investigators. Metabolism 45:699-706

7. Ng MC, So WY, Lam VK et al (2004) Genome-wide scan for metabolic syndrome and related quantitative traits in Hong Kong Chinese and confirmation of a susceptibility locus on chromosome 1q21-q25. Diabetes 53:2676-2683

8. Rich SS, Bowden DW, Haffner SM et al (2004) Identification of quantitative trait loci for glucose homeostasis: the Insulin Resistance Atherosclerosis Study (IRAS) Family Study. Diabetes 53:1866-1875

9. Chiu YF, Chuang LM, Hsiao CF et al (2005) An autosomal genomewide scan for loci linked to pre-diabetic phenotypes in nondiabetic Chinese subjects from the Stanford Asia-Pacific Program of Hypertension and Insulin Resistance Family Study. Diabetes 54:1200-1206

10. Samani NJ (2003) Genome scans for hypertension and blood pressure regulation. Am J Hypertens 16:167-171

11. McQueen MB, Bertram L, Rimm EB, Blacker D, Santangelo SL (2003) A QTL genome scan of the metabolic syndrome and its component traits. BMC Genet 4(Suppl 1):S96

12. Glazier AM, Nadeau JH, Aitman TJ (2002) Finding genes that underlie complex traits. Science 298:2345-2349

13. Province MA, Kardia SL, Ranade K et al (2003) A meta-analysis of genome-wide linkage scans for hypertension: the National
Heart, Lung and Blood Institute Family Blood Pressure Program. Am J Hypertens 16:144-147

14. Loos RJ, Katzmarzyk PT, Rao DC et al (2003) Genome-wide linkage scan for the metabolic syndrome in the HERITAGE Family Study. J Clin Endocrinol Metab 88:5935-5943

15. Hamet P, Merlo E, Seda O et al (2005) Quantitative founder-effect analysis of French Canadian families identifies specific loci contributing to metabolic phenotypes of hypertension. Am J Hum Genet 76:815-832

16. Kraja AT, Rao DC, Weder AB et al (2005) Two major QTLs and several others relate to factors of metabolic syndrome in the Family Blood Pressure Program. Hypertension 46:751-757

17. Wu KD, Hsiao CF, Ho LT et al (2002) Clustering and heritability of insulin resistance in Chinese and Japanese hypertensive families: a Stanford-Asian Pacific Program in Hypertension and Insulin Resistance sibling study. Hypertens Res 25:529-536

18. Kraja AT, Rao DC, Weder AB et al (2005) An evaluation of the metabolic syndrome in a large multi-ethnic study: the Family Blood Pressure Program. Nutr Metab (Lond) 2:17

19. Lehman DM, Arya R, Blangero J et al (2005) Bivariate linkage analysis of the insulin resistance syndrome phenotypes on chromosome 7q. Hum Biol 77:231-246

20. Yang WS, Hsiung CA, Ho LT et al (2003) Genetic epistasis of adiponectin and PPARgamma2 genotypes in modulation of insulin sensitivity: a family-based association study. Diabetologia 46:977-983

21. Ranade $\mathrm{K}, \mathrm{Wu} \mathrm{KD}$, Risch $\mathrm{N}$ et al (2001) Genetic variation in aldosterone synthase predicts plasma glucose levels. Proc Natl Acad Sci USA 98:13219-13224

22. Ranade K, Hinds D, Hsiung CA et al (2003) A genome scan for hypertension susceptibility loci in populations of Chinese and Japanese origins. Am J Hypertens 16:158-162

23. Blangero J, Williams JT, Almasy L (2001) Variance component methods for detecting complex trait loci. Adv Genet 42:151181

24. Hwu CM, Hsiao CF, Kuo SW et al (2004) Physical inactivity is an important lifestyle determinant of insulin resistance in hypertensive patients. Blood Press 13:355-361

25. Blangero J (1995) Genetic analysis of a common oligogenic trait with quantitative correlates: summary of GAW9 results. Genet Epidemiol 12:689-706

26. Almasy L, Blangero J (1998) Multipoint quantitative-trait linkage analysis in general pedigrees. Am J Hum Genet 62:1198-1211

27. Amos C, de Andrade M, Zhu D (2001) Comparison of multivariate tests for genetic linkage. Hum Hered 51:133-144

28. Turner ST, Kardia SL, Boerwinkle E, Andrade MdM (2004) Multivariate linkage analysis of blood pressure and body mass index. Genet Epidemiol 27:64-73

29. Almasy L, Dyer TD, Blangero J (1997) Bivariate quantitative trait linkage analysis: pleiotropy vs co-incident linkages. Genet Epidemiol 14:953-958

30. Arya R, Blangero J, Williams K et al (2002) Factors of insulin resistance syndrome-related phenotypes are linked to genetic locations on chromosomes 6 and 7 in nondiabetic MexicanAmericans. Diabetes 51:841-847

31. Evans DM (2002) The power of multivariate quantitative-trait loci linkage analysis is influenced by the correlation between variables. Am J Hum Genet 70:1599-1602

32. Yoshida H, Ohagi S, Sanke T, Furuta H, Furuta M, Nanjo K (1995) Association of the prohormone convertase 2 gene (PCSK2) on chromosome 20 with NIDDM in Japanese subjects. Diabetes 44:389-393

33. Hsiao C-F, Chiu Y-F, Chiang F-T et al, The SAPPHIRe Study Group (2006) Genome-wide linkage analysis of lipids in Chinese and Japanese from the SAPPHIRe Family Study. Am J Hypertens 19:1270-1277 
34. Duggirala R, Blangero J, Almasy L et al (2001) A major locus for fasting insulin concentrations and insulin resistance on chromosome $6 \mathrm{q}$ with strong pleiotropic effects on obesity-related phenotypes in nondiabetic Mexican Americans. Am J Hum Genet 68:1149-1164

35. Wang X, Paigen B (2005) Genetics of variation in HDL cholesterol in humans and mice. Circ Res 96:27-42

36. Almasy L, Hixson JE, Rainwater DL et al (1999) Human pedigree-based quantitative-trait-locus mapping: localization of two genes influencing HDL-cholesterol metabolism. Am J Hum Genet 64:1686-1693
37. Lambert G, Amar MJ, Guo G, Brewer HB Jr, Gonzalez FJ, Sinal CJ (2003) The farnesoid X-receptor is an essential regulator of cholesterol homeostasis. J Biol Chem 278:2563-2570

38. Langefeld CD, Wagenknecht LE, Rotter JI et al (2004) Linkage of the metabolic syndrome to 1q23-q31 in Hispanic families: the Insulin Resistance Atherosclerosis Study Family Study. Diabetes 53:1170-1174

39. Rich SS, Bowden DW, Haffner SM et al (2005) A genome scan for fasting insulin and fasting glucose identifies a quantitative trait locus on chromosome 17p: the insulin resistance atherosclerosis study (IRAS) family study. Diabetes 54:290-295 